\title{
On $\phi$-contractions in partially ordered fuzzy metric spaces
}

\section{Shuang Wang*}

\section{"Correspondence:}

wangshuang19841119@163.com School of Mathematical Sciences, Yancheng Teachers University, Yancheng, Jiangsu 224051, P.R. China

\section{Springer}

\begin{abstract}
Under some weaker conditions, some coincidence point and common fixed point theorems are established in partially ordered fuzzy metric spaces using weakly compatible mappings. By using the theorems, we obtain some coupled and multidimensional fixed point results, which are generalization and improvement of very recent theorems in the corresponding literature. In order to illustrate our main results, we give three examples.
\end{abstract}

MSC: $47 \mathrm{H} 10 ; 54 \mathrm{H} 25$

Keywords: fuzzy metric spaces; weakly compatible mappings; common fixed points; coupled coincidence points; multidimensional coincidence points; partially ordered

\section{Introduction}

In 1987, the notion of coupled fixed point was first introduced by Guo and Lakshmikantham [1]. Recently, Gnana-Bhaskar and Lakshmikantham [2] established some coupled fixed point theorems in partially ordered metric space. The fuzzy version of the results of Gnana-Bhaskar and Lakshmikantham [2] was studied by Sedghi et al. [3]. After that, common coupled fixed point results in fuzzy metric spaces were established by $\mathrm{Hu}[4]$ and Hu et al. [5]. Very recently, Choudhury et al. [6] established coupled coincidence point and fixed point results for compatible mappings in partially ordered fuzzy metric spaces. Later, Roldán et al. [7] obtained multidimensional coincidence point theorems for nonlinear mappings in any number of variables in partially ordered fuzzy metric spaces. Their results generalize, clarify and unify several classical and very recent related results in the literature in the setting of metric spaces.

But many results (see, e.g., [4-7]) are obtained under the assumptions: (a) $\phi(t)=k t$ for all $t>0$, where $k \in(0,1)$; or (b) $\sum_{n=1}^{\infty} \phi^{n}(t)<\infty$ for all $t>0$. It is obvious that the condition (a) is special. In [8], Cirić [8] has pointed out, the condition (b) is very strong and difficult for testing in practice. Then Ćirić introduced the condition (CBW): $\phi(0)=0, \phi(t)<t$ and $\liminf _{r \rightarrow t^{+}} \phi(t)<t$ for all $t>0$. Later, Jachymski [9] presented the condition (c): $0<\phi(t)<t$ and $\lim _{n \rightarrow \infty} \phi^{n}(t)=0$ for all $t>0$. In order to weaken the condition (c) further, Fang [10] introduced the condition (d): for each $t>0$ there exists $r \geq t$ such that $\lim _{n \rightarrow \infty} \phi^{n}(r)=$ 0 in the context of Menger probabilistic metric spaces and fuzzy metric spaces. In this paper, under the condition (d), we present some coincidence point and common fixed

(c) 2015 Wang. This article is distributed under the terms of the Creative Commons Attribution 4.0 International License (http://creativecommons.org/licenses/by/4.0/), which permits unrestricted use, distribution, and reproduction in any medium, provided you give appropriate credit to the original author(s) and the source, provide a link to the Creative Commons license, and indicate if changes were made. 
point results for weakly compatible mappings in partially ordered fuzzy metric spaces. By using the theorems, we obtain some coupled and multidimensional fixed point results, which are generalization and improvement of very recent theorems in the corresponding literature. In addition, we illustrate our main results with three examples.

\section{Preliminaries}

In order to fix the framework needed to state our main results, we recall the following notions. Let $n \in \mathbb{N}, X$ be a non-empty set and $X^{n}$ be the Cartesian product of $n$ copies of $X$. For brevity, $g(x),\left(y_{1}, y_{2}, \ldots, y_{n}\right),\left(y_{m}^{1}, y_{m}^{2}, \ldots, y_{m}^{n}\right),\left(z_{m}^{1}, z_{m}^{2}, \ldots, z_{m}^{n}\right),\left(z_{1}, z_{2}, \ldots, z_{n}\right),\left(v_{1}, v_{2}, \ldots, v_{n}\right)$ and $\left(x_{0}^{1}, x_{0}^{2}, \ldots, x_{0}^{n}\right)$ will be denoted by $g x, Y, Y_{m}, Z_{m}, Z, V$, and $X_{0}$, respectively.

Throughout this paper, let $\{A, B\}$ be a partition of the set $\Lambda_{n}=\{1,2, \ldots, n\}$, i.e., $A \cup B=$ $\Lambda_{n}$ and $A \cap B=\emptyset$. Let $\sigma_{1}, \sigma_{2}, \ldots, \sigma_{n}: \Lambda_{n} \rightarrow \Lambda_{n}$ be $n$ mappings from $\Lambda_{n}$ into itself. We denote $\Omega_{A, B}=\left\{\sigma: \Lambda_{n} \rightarrow \Lambda_{n}: \sigma(A) \subseteq A\right.$ and $\left.\sigma(B) \subseteq B\right\}, \Omega_{A, B}^{\prime}=\left\{\sigma: \Lambda_{n} \rightarrow \Lambda_{n}: \sigma(A) \subseteq\right.$ $B$ and $\sigma(B) \subseteq A\}, \mathbb{N}_{0}=\{0,1, \ldots, n, \ldots\}, \mathbb{N}=\{1, \ldots, n, \ldots\}, \mathbb{R}^{+}=[0, \infty)$, and $\mathbb{I}=[0,1]$. If $(X, \preceq)$ is a partially ordered space, we use the following notation from [11], for $y, v \in X$ and $i \in \Lambda_{n}$

$$
y \preceq_{i} v \Leftrightarrow \begin{cases}y \preceq v, & \text { if } i \in A, \\ y \succeq v, & \text { if } i \in B .\end{cases}
$$

Consider on $X^{n}$ the next natural partial order: for $Y, V \in X^{n}$

$$
Y \preceq_{n} V \quad \Leftrightarrow \quad y_{i} \preceq_{i} v_{i}
$$

for all $i$. If $Y \preceq_{n} V$ or $Y \succeq_{n} V$, then two points $Y$ and $V$ are comparable (denoted by $Y \asymp V$ ).

Proposition 2.1 ([12]) If $Y \preceq_{n} V$, it follows that $\left(y_{\sigma(1)}, y_{\sigma(2)}, \ldots, y_{\sigma(n)}\right) \preceq_{n}\left(v_{\sigma(1)}, v_{\sigma(2)}, \ldots\right.$, $\left.v_{\sigma(n)}\right)$ if $\sigma \in \Omega_{A B},\left(y_{\sigma(1)}, y_{\sigma(2)}, \ldots, y_{\sigma(n)}\right) \succeq_{n}\left(v_{\sigma(1)}, v_{\sigma(2)}, \ldots, v_{\sigma(n)}\right)$ if $\sigma \in \Omega_{A B}^{\prime}$.

Definition 2.2 ([13]) Let $\left(X^{n}, \preceq_{n}\right)$ be a partially ordered set, and $T$ and $G$ be self-mappings of $X^{n}$. It is said that $T$ is a $G$-isotone mapping if, for any $Y_{1}, Y_{2} \in X^{n}$

$$
G\left(Y_{1}\right) \preceq_{n} G\left(Y_{2}\right) \quad \Rightarrow \quad T\left(Y_{1}\right) \preceq_{n} T\left(Y_{2}\right) .
$$

Definition 2.3 ([14]) Let $(X, \preceq)$ be a partially ordered set and $F: X^{2} \rightarrow X$. We say $F$ has the mixed $g$-monotone property if $F$ is monotone non-decreasing in its first argument and is monotone non-increasing in its second argument, that is, for any $x, y \in X$,

$$
x_{1}, x_{2} \in X, \quad g\left(x_{1}\right) \preceq g\left(x_{2}\right) \quad \text { implies } \quad q F\left(x_{1}, y\right) \preceq F\left(x_{2}, y\right),
$$

and

$y_{1}, y_{2} \in X, \quad g\left(y_{1}\right) \preceq g\left(y_{2}\right) \quad$ implies $\quad F\left(x, y_{1}\right) \succeq F\left(x, y_{2}\right)$.

Definition 2.4 ([11]) Let $(X, \preceq)$ be a partially ordered space. We say that $F$ has the mixed gmonotone property if $F$ is $g$-monotone non-decreasing in arguments of $A$ and $g$-monotone non-increasing in arguments of $B$, i.e., for all $x_{1}, x_{2}, \ldots, x_{n}, y, z \in X$ and all $i$

$$
g y \preceq g z \quad \Rightarrow \quad F\left(x_{1}, \ldots, x_{i-1}, y, x_{i+1}, \ldots, x_{n}\right) \preceq_{i} F\left(x_{1}, \ldots, x_{i-1}, z, x_{i+1}, \ldots, x_{n}\right) .
$$


Definition 2.5 ([13]) An element $Y \in X^{n}$ is called a coincidence point of the mappings $T: X^{n} \rightarrow X^{n}$ and $G: X^{n} \rightarrow X^{n}$ if $T(Y)=G(Y)$. Furthermore, if $T(Y)=G(Y)=Y$, then we say that $Y$ is a common fixed point of $T$ and $G$.

Definition 2.6 Let $F: X^{n} \rightarrow X$ and $g: X \rightarrow X$ be two mappings. A point $\left(x_{1}, x_{2}, \ldots, x_{n}\right) \in$ $X^{n}$ is:

(i) A coupled coincidence point ([14]) if $n=2, F\left(x_{1}, x_{2}\right)=g\left(x_{1}\right)$, and $F\left(x_{2}, x_{1}\right)=g\left(x_{2}\right)$. If $g$ is the identity mapping on $X$, then $\left(x_{1}, x_{2}\right) \in X^{2}$ is called a coupled fixed point of the mapping $F([2])$. A coupled common fixed point of $F$ and $g$ ([15]) if $n=2$, $F\left(x_{1}, x_{2}\right)=g\left(x_{1}\right)=x_{1}$, and $F\left(x_{2}, x_{1}\right)=g\left(x_{2}\right)=x_{2}$.

(ii) A $\Upsilon$-coincidence point ([16]) of $F$ and $g$ if

$$
F\left(x_{\sigma_{i}(1)}, x_{\sigma_{i}(2)}, \ldots, x_{\sigma_{i}(n)}\right)=g x_{i}
$$

for $i \in \Lambda_{n}$. If $g$ is the identity mapping on $X$, then $\left(x_{1}, x_{2}, \ldots, x_{n}\right) \in X^{n}$ is called a $\Upsilon$-fixed point of the mapping $F$.

Definition 2.7 ([17]) A triple $(X, \tau, \preceq)$ is called a partially ordered topological space if $\tau$ is a Hausdorff topology on $X$ and $\preceq$ is a partial order on $X$. A partially ordered topological space $(X, \tau, \preceq)$ is said to have the sequential $g$-monotone property if it verifies:

(i) If $\left\{x_{m}\right\}$ is a non-decreasing sequence and $\left\{x_{m}\right\} \rightarrow x$, then $g x_{m} \preceq g x$ for all $m$.

(ii) If $\left\{y_{m}\right\}$ is a non-increasing sequence and $\left\{y_{m}\right\} \rightarrow y$, then $g y_{m} \succeq g y$ for all $m$.

If $g$ is the identity mapping, then $X$ is said to have the sequential monotone property.

Definition 2.8 ([18]) A triangular norm (also called a $t$-norm) is a map $*: \mathbb{I} \times \mathbb{I} \rightarrow \mathbb{I}$ that is associative, commutative, non-decreasing in both arguments and has 1 as identity. A $t$-norm is continuous if it is continuous in $\mathbb{I}^{2}$ as mapping. If $a_{1}, a_{2}, \ldots, a_{m} \in \mathbb{I}$, then

$$
*_{i=1}^{m} a_{i}=a_{1} * a_{2} * \cdots * a_{m}
$$

For each $a \in[0,1]$, the sequence $\left\{*^{m} a\right\}_{m=1}^{\infty}$ is defined inductively by $*^{1} a=a$ and $*^{m+1} a=$ $\left(*^{m} a\right) * a$ for all $m \geq 1$.

Definition 2.9 ([19]) A $t$-norm is said to be of $H$-type if the sequence $\left\{*^{m} a\right\}_{m=1}^{\infty}$ is equicontinuous at $a=1$, i.e., for all $\varepsilon \in(0,1)$, there exists $\eta \in(0,1)$ such that if $a \in(1-\eta, 1]$, then $*^{m} a>1-\varepsilon$ for all $m \in \mathbb{N}$.

Definition 2.10 ([20]) A fuzzy metric space in the sense of Kramosil and Michálek (briefly, a $F M S)$ is a triple $(X, M, *)$ where $X$ is a non-empty set, $*$ is a $t$-norm and $M: X \times X \times \mathbb{R}^{+} \rightarrow \mathbb{I}$ is a fuzzy set satisfying the following conditions for all $x, y, z \in X$ and $t, s \geq 0$ :

$(\mathrm{FM}-1) \quad M(x, y, 0)=0$

(FM-2) $M(x, y, t)=1$, for all $t>0$ if and only if $x=y$;

(FM-3) $M(x, y, t)=M(y, x, t)$;

$(\mathrm{FM}-4) \quad M(x, y, t) * M(y, z, s) \leq M(x, z, t+s)$;

$\left(\right.$ FM-5) $M(x, y, \cdot): \mathbb{R}^{+} \rightarrow \mathbb{I}$ is left continuous.

Remark 2.11 Note that $*$ is continuous in the original definition in [20]. 
Definition $2.12([21])$ A triple $(X, M, *)$ is called a fuzzy metric space (in the sense of George and Veeramani) if $X$ is an arbitrary non-empty set, $*$ is a continuous $t$-norm and $M: X \times X \times \mathbb{R}^{+} \rightarrow \mathbb{I}$ is a fuzzy set satisfying, for each $x, y, z \in X$ and $t, s>0$, conditions (FM-2), (FM-3), (FM-4), (GV-5): $M(x, y, \cdot):(0, \infty) \rightarrow \mathbb{I}$ is continuous, and (GV-1): $M(x, y, t)>0$.

Definition 2.13 ([21]) Let $(X, M, *)$ be a $F M S$. A sequence $\left\{x_{n}\right\}$ in $X$ is said to be convergent to $x \in X$ if $\lim _{n \rightarrow \infty} M\left(x_{n}, x, t\right)=1$ for all $t>0$. A sequence $\left\{x_{n}\right\}$ in $X$ is said to an $M$-Cauchy sequence, if for each $\varepsilon \in(0,1)$ and $t>0$ there exists $n_{0} \in \mathbb{N}$ such that $M\left(x_{n}, x_{m}, t\right)>1-\varepsilon$ for all $m, n \geq n_{0}$. A fuzzy metric space is called complete if every $M$-Cauchy sequence is convergent in $X$.

Lemma 2.14 ([22]) If $(X, M)$ is a FMS under some $t$-norm and $x, y \in X$, then $M(x, y, \cdot)$ is a non-decreasing function on $(0, \infty)$.

Definition 2.15 ([7]) A partially ordered fuzzy metric space (for short, poFMS) is a quadruple $(X, M, *, \preceq)$ such that $(X, M, *)$ is a $F M S$ and $\preceq$ is a partial order on $X$.

Definition 2.16 ([7]) Let $p \in \mathbb{N}$ and let $(X, M, *)$ be a $F M S$. A mapping $G: X^{p} \rightarrow X$ is said to be continuous at a point $Y_{0} \in X^{p}$ if, for any sequence $\left\{Y_{m}\right\}_{m \geq 0}$ in $X^{p}$ converging to $Y_{0}$, the sequence $\left\{G\left(Y_{m}\right)\right\}_{m \geq 0}$ converges to $G\left(Y_{0}\right)$. If $G$ is continuous at each $Y_{0} \in X^{p}$, then $G$ is said continuous on $X^{p}$.

Definition 2.17 ([4]) Let $(X, M, *)$ be a $F M S$. The mappings $F$ and $g$ where $F: X^{2} \rightarrow X$ and $g: X \rightarrow X$, are said to be compatible if for all $t>0$

$$
\lim _{n \rightarrow \infty} M\left(g\left(F\left(x_{n}, y_{n}\right)\right), F\left(g\left(x_{n}\right), g\left(y_{n}\right)\right), t\right)=1
$$

and

$$
\lim _{n \rightarrow \infty} M\left(g\left(F\left(y_{n}, x_{n}\right)\right), F\left(g\left(y_{n}\right), g\left(x_{n}\right)\right), t\right)=1,
$$

whenever $\left\{x_{n}\right\}$ and $\left\{y_{n}\right\}$ are sequences in $X$ such that $\lim _{n \rightarrow \infty} F\left(x_{n}, y_{n}\right)=\lim _{n \rightarrow \infty} g\left(x_{n}\right)=x$ and $\lim _{n \rightarrow \infty} F\left(y_{n}, x_{n}\right)=\lim _{n \rightarrow \infty} g\left(y_{n}\right)=y$ for some $x, y \in X$.

Definition 2.18 ([7]) Let $(X, M, *, \preceq)$ be a poFMS and let $\Phi=\left(\sigma_{1}, \sigma_{2}, \ldots, \sigma_{n}\right)$ be an $n$ tuple of mappings from $\Lambda_{n}$ into itself. Two mappings $F: X^{n} \rightarrow X$ and $g: X \rightarrow X$ are said to be $\Phi$-compatible if, for all sequences $\left\{x_{m}^{1}\right\}_{m \geq 0},\left\{x_{m}^{2}\right\}_{m \geq 0}, \ldots,\left\{x_{m}^{n}\right\}_{m \geq 0} \subset X$ such that $\left\{g x_{m}^{1}\right\}_{m \geq 0},\left\{g x_{m}^{2}\right\}_{m \geq 0}, \ldots,\left\{g x_{m}^{n}\right\}_{m \geq 0}$ are monotone and

$$
\exists \lim _{m \rightarrow \infty} F\left(x_{m}^{\sigma_{i}(1)}, x_{m}^{\sigma_{i}(2)}, \ldots, x_{m}^{\sigma_{i}(n)}\right)=\lim _{m \rightarrow \infty} g x_{m}^{i} \in X \quad \text { for all } i,
$$

we have

$$
\lim _{m \rightarrow \infty} M\left(g F\left(x_{m}^{\sigma_{i}(1)}, x_{m}^{\sigma_{i}(2)}, \ldots, x_{m}^{\sigma_{i}(n)}\right), F\left(g x_{m}^{\sigma_{i}(1)}, g x_{m}^{\sigma_{i}(2)}, \ldots, g x_{m}^{\sigma_{i}(n)}\right), t\right)=1
$$

for all $t>0$ and all $i$. 
Remark 2.19 If $n=1$ in Definition 2.18, then $F, g: X \rightarrow X$ are compatible w.r.t. ( $X, M$, $*, \preceq)$.

Definition 2.20 ([23]) We will say that the maps $f, g: X \rightarrow X$ are weakly compatible (or the pair $(f, g)$ is $w$-compatible) if $f g x=g f x$ for all $x \in X$ such that $f x=g x$.

Let $\Phi^{\prime}$ denote the family of all functions $\phi: \mathbb{R}^{+} \rightarrow \mathbb{R}^{+}$such that $\lim _{n \rightarrow \infty} \phi^{n}(t)=0$ for all $t>0$, and let $\Phi_{w}$ denote the family of all functions $\phi: \mathbb{R}^{+} \rightarrow \mathbb{R}^{+}$verifying the condition (d), that is, for each $t>0$ there exists $r \geq t$ such that $\lim _{n \rightarrow \infty} \phi^{n}(r)=0$.

It is evident that the condition $\lim _{n \rightarrow \infty} \phi^{n}(t)=0$ for all $t>0$ implies the condition (d). However, the following example shows that the reverse is not true in general. Hence $\Phi^{\prime} \subseteq \Phi_{w}$

Example 2.21 ([10]) Let the function $\phi: \mathbb{R}^{+} \rightarrow \mathbb{R}^{+}$be defined by

$$
\phi(t)= \begin{cases}\frac{t}{1+t}, & \text { if } 0 \leq t<1 \\ -\frac{t}{3}+\frac{4}{3}, & \text { if } 1 \leq t \leq 2, \\ t-\frac{4}{3}, & \text { if } 2<t<\infty .\end{cases}
$$

Notice that $\phi \in \Phi_{w}$ but $\phi \notin \Phi^{\prime}$.

Lemma 2.22 ([10]) Let $\phi \in \Phi_{w}$, then for each $t>0$ there exists $r \geq t$ such that $\phi(r)<t$.

\section{Main results}

In this section we establish our main results and use them to obtain some coupled and multidimensional fixed point theorems.

Lemma 3.1 If $(X, M, *)$ is a FMS with $M(x, y, \cdot): \mathbb{R}^{+} \rightarrow \mathbb{I}$ is continuous, then $M$ is a continuous mapping on $X^{2} \times(0, \infty)$.

Proof The proof is the same as that for a fuzzy metric space in the sense of George and Veeramani (see Rodríguez-López and Romaguera [24], Proposition 1).

Lemma 3.2 Let $(X, M, *)$ be a FMS such that $*$ is a t-norm of H-type. Let $\left\{x_{n}\right\}$ be a sequence in $(X, M, *)$. If there exists a function $\phi \in \Phi_{w}$ satisfying

(i) $\phi(t)>0$ for all $t>0$;

(ii) $M\left(x_{n}, x_{m}, \phi(t)\right) \geq M\left(x_{n-1}, x_{m-1}, t\right)$ for all $n, m \in \mathbb{N}$ and $t>0$;

(iii) $\lim _{t \rightarrow \infty} M\left(x_{0}, x_{1}, t\right)=1$,

then $\left\{x_{m}\right\}$ is a Cauchy sequence.

Proof We proceed with the following steps:

Step 1 . We claim that for any $t>0$,

$$
M\left(x_{n}, x_{n+1}, t\right) \rightarrow 1 \quad \text { as } n \rightarrow \infty .
$$

By (iii), for any $\varepsilon \in(0,1)$, there exists $t_{0}>0$ such that $M\left(x_{0}, x_{1}, t_{0}\right)>1-\varepsilon$. Since $\phi \in \Phi_{w}$, there exists $t_{1} \geq t_{0}$ such that $\lim _{n \rightarrow \infty} \phi^{n}\left(t_{1}\right)=0$. Thus, for each $t>0$, there exists $n_{0} \in \mathbb{N}$ 
such that $\phi^{n}\left(t_{1}\right)<t$ for all $n \geq n_{0}$. It is evident that (ii) implies that

$$
M\left(x_{n}, x_{n+1}, \phi(t)\right) \geq M\left(x_{n-1}, x_{n}, t\right) \quad \text { for all } n \in \mathbb{N} \text { and } t>0
$$

It follows from (i) that $\phi^{n}(t)>0$ for all $n \in \mathbb{N}$ and $t>0$. By induction, it follows from (4) that

$$
M\left(x_{n}, x_{n+1}, \phi^{n}(t)\right) \geq M\left(x_{0}, x_{1}, t\right) \quad \text { for all } n \in \mathbb{N} \text { and } t>0 \text {. }
$$

So, by (5) and the monotonicity of $M(x, y, \cdot)$, we have

$$
M\left(x_{n}, x_{n+1}, t\right) \geq M\left(x_{n}, x_{n+1}, \phi^{n}\left(t_{1}\right)\right) \geq M\left(x_{0}, x_{1}, t_{1}\right) \geq M\left(x_{0}, x_{1}, t_{0}\right)>1-\varepsilon
$$

for all $n \geq n_{0}$. Taking into account that $\varepsilon, t>0$ are arbitrary, we conclude that (3) holds.

Step 2 . We claim that for any $t>0$,

$$
M\left(x_{n}, x_{m}, t\right) \geq *^{m-n} M\left(x_{n}, x_{n+1}, t-\phi(r)\right) \quad \text { for all } m \geq n+1
$$

where $r \geq t$. Since $\phi \in \Phi_{w}$, for any $t>0$, there exists $r \geq t$ such that $\phi(r)<t$ by Lemma 2.22. Since $M\left(x_{n}, x_{n+1}, t\right) \geq M\left(x_{n}, x_{n+1}, t-\phi(r)\right)=*^{1} M\left(x_{n}, x_{n+1}, t-\phi(r)\right)$, then (6) holds for $m=$ $n+1$. Suppose now that $M\left(x_{n}, x_{m}, t\right) \geq *^{m-n} M\left(x_{n}, x_{n+1}, t-\phi(r)\right)$ holds for some fixed $m \geq$ $n+1$. By (FM-4), (ii) and the monotonicity of $*$, we get

$$
\begin{aligned}
M\left(x_{n}, x_{m+1}, t\right) & =M\left(x_{n}, x_{m+1}, t-\phi(r)+\phi(r)\right) \\
& \geq M\left(x_{n}, x_{n+1}, t-\phi(r)\right) * M\left(x_{n+1}, x_{m+1}, \phi(r)\right) \\
& \geq M\left(x_{n}, x_{n+1}, t-\phi(r)\right) * M\left(x_{n}, x_{m}, r\right) \\
& \geq M\left(x_{n}, x_{n+1}, t-\phi(r)\right) * M\left(x_{n}, x_{m}, t\right) \\
& \geq M\left(x_{n}, x_{n+1}, t-\phi(r)\right) *\left(*^{m-n} M\left(x_{n}, x_{n+1}, t-\phi(r)\right)\right) \\
& =*^{m+1-n} M\left(x_{n}, x_{n+1}, t-\phi(r)\right) .
\end{aligned}
$$

Thus, we prove that if (6) holds for some $m \geq n+1$, then it also holds for $m+1$. By induction, we conclude that (6) holds for all $m \geq n+1$.

Step 3. We claim that $\left\{x_{n}\right\}$ is a Cauchy sequence. As $*$ is a $t$-norm of H-type, for any $\varepsilon \in(0,1)$ there exists $\eta \in(0,1)$ such that

$$
\text { if } a \in(1-\eta, 1], \quad \text { then } *^{l} a>1-\varepsilon \quad \text { for all } l \in \mathbb{N} \text {. }
$$

It follows from (3) that there exists $n_{1} \in \mathbb{N}$ such that $M\left(x_{n}, x_{n+1}, t-\phi(r)\right)>1-\eta$ for all $n \geq n_{1}$. So, by (7), we have

$$
*^{m-n} M\left(x_{n}, x_{n+1}, t-\phi(r)\right)>1-\varepsilon
$$

for all $m>n \geq n_{1}$. By (6) and (8), we get for each $t>0$ and $\varepsilon \in(0,1), M\left(x_{n}, x_{m}, t\right)>1-\varepsilon$ for all $m>n \geq n_{1}$, which implies that $\left\{x_{n}\right\}$ is a Cauchy sequence. 
Now, we state and prove some fixed point results for weakly compatible mappings in partially ordered fuzzy metric spaces.

Theorem 3.3 Let $(X, M, *, \preceq)$ be a complete poFMS such that $*$ is a t-norm of H-type. Let $T: X \rightarrow X$ and $G: X \rightarrow X$ be two mappings such that $T$ is a G-isotone mapping and $T(X) \subseteq G(X)$. Assume that there exists $\phi \in \Phi_{w}$ such that, for all $t>0$ and $y, v \in X$ with $G(y) \preceq G(v)$,

$$
M(T(y), T(v), \phi(t)) \geq M(G(y), G(v), t) .
$$

\section{Also suppose that either}

(C1) $T$ and $G$ are continuous and compatible and $M(x, y, \cdot): \mathbb{R}^{+} \rightarrow \mathbb{I}$ is continuous or

(C2) $\left(X, \tau_{M}, \preceq\right)$ has the sequential monotone property and $G(X)$ is closed.

If there exists $y_{0} \in X$ such that $G\left(y_{0}\right) \asymp T\left(y_{0}\right)$ and $\lim _{t \rightarrow \infty} M\left(G\left(y_{0}\right), T\left(y_{0}\right), t\right)=1$. Then $T$ and $G$ have a coincidence point.

Proof Let $y_{0} \in X$ such that $G\left(y_{0}\right) \asymp T\left(y_{0}\right)$ and $\lim _{t \rightarrow \infty} M\left(G\left(y_{0}\right), T\left(y_{0}\right), t\right)=1$. Since $T(X) \subseteq$ $G(X)$, there exists $y_{1} \in X$ such that $G\left(y_{1}\right)=T\left(y_{0}\right)$. Recursively, we see that, for every $m \in \mathbb{N}_{0}$, there exists $y_{m+1} \in X$ such that $G\left(y_{m+1}\right)=T\left(y_{m}\right)$. Set $z_{0}=G\left(y_{0}\right)$ and $z_{m+1}=G\left(y_{m+1}\right)=T\left(y_{m}\right)$ for every $m \in \mathbb{N}_{0}$.

Since $G\left(y_{0}\right) \asymp T\left(y_{0}\right)$, we suppose that $G\left(y_{0}\right) \preceq T\left(y_{0}\right)$, i.e., $z_{0} \preceq z_{1}$ (the case $G\left(y_{0}\right) \succeq T\left(y_{0}\right)$ is treated similarly). Assume that $z_{m-1} \preceq z_{m}$ for some $m \in \mathbb{N}_{0}$, that is, $G\left(y_{m-1}\right) \preceq G\left(y_{m}\right)$. Since $T$ is a $G$-isotone mapping, we get $z_{m}=T\left(y_{m-1}\right) \preceq T\left(y_{m}\right)=z_{m+1}$. This actually means that the sequence $\left\{z_{m}\right\}$ is non-decreasing. Using (9) and monotonicity of $\left\{z_{m}\right\}$, we get

$$
\begin{aligned}
M\left(z_{n}, z_{m}, \phi(t)\right) & =M\left(T\left(y_{n-1}\right), T\left(y_{m-1}\right), \phi(t)\right) \\
& \geq M\left(G\left(y_{n-1}\right), G\left(y_{m-1}\right), t\right) \\
& =M\left(z_{n-1}, z_{m-1}, t\right)
\end{aligned}
$$

for all $m, n \in \mathbb{N}$ and $t>0$. Obviously, the inequality (9) implies that $\phi(t)>0$ for all $t>0$. Indeed, if there exists some $t_{0}>0$ such that $\phi\left(t_{0}\right)=0$. It follows from (9) that

$$
0=M\left(T(y), T(y), \phi\left(t_{0}\right)\right) \geq M\left(G(y), G(y), t_{0}\right)=1,
$$

which is a contradiction. Since $\lim _{t \rightarrow \infty} M\left(G\left(y_{0}\right), T\left(y_{0}\right), t\right)=1$, we have $\lim _{t \rightarrow \infty} M\left(z_{0}, z_{1}\right.$, $t)=1$. So, by Lemma 3.2, $\left\{z_{m}\right\}$ is a Cauchy sequence.

Now suppose that the condition $(\mathrm{C} 1)$ holds. Since $(X, M, *, \preceq)$ is complete, there exists $\hat{z} \in X$ such that $\lim _{m \rightarrow \infty} z_{m}=\hat{z}$, that is,

$$
\lim _{m \rightarrow \infty} T\left(y_{m}\right)=\lim _{m \rightarrow \infty} G\left(y_{m}\right)=\hat{z}
$$

Since $T$ and $G$ are compatible, we have

$$
\lim _{m \rightarrow \infty} M\left(G\left(G\left(y_{m+1}\right)\right), T\left(G\left(y_{m}\right)\right), t\right)=\lim _{m \rightarrow \infty} M\left(G\left(T\left(y_{m}\right)\right), T\left(G\left(y_{m}\right)\right), t\right)=1
$$


for all $t>0$. As $G$ is continuous, we have

$$
\lim _{m \rightarrow \infty} G\left(G\left(y_{m}\right)\right)=G(\hat{z})
$$

Using Lemma 3.1, we find that $M$ is a continuous mapping on $X^{2} \times(0, \infty)$. By the continuity of $M$ and (10)-(12), we have $1=\lim _{m \rightarrow \infty} M\left(G\left(G\left(y_{m+1}\right)\right), T\left(G\left(y_{m}\right)\right), t\right)=M(G(\hat{z}), T(\hat{z}), t)$ for all $t>0$, which implies that $G(\hat{z})=T(\hat{z})$ and $\hat{z}$ is a coincidence point of $T$ and $G$.

Now suppose that the condition (C2) holds. Since $(X, M, *, \preceq)$ is complete and $G(X)$ is closed, there exists $z \in X$ such that $\lim _{m \rightarrow \infty} T\left(y_{m}\right)=\lim _{m \rightarrow \infty} G\left(y_{m}\right)=G(z)$. Since $\left(X, \tau_{M}, \preceq\right)$ has the sequential monotone property, we have $G\left(y_{m}\right) \preceq G(z)$ for all $m \in \mathbb{N}_{0}$. Since $\phi \in$ $\Phi_{w}$, for each $t>0$ there exists $r \geq t$ such that $\phi(r)<t$ by Lemma 2.22. So, by (9) and the monotonicity of $M(x, y, \cdot)$, we have

$$
M\left(T\left(y_{m}\right), T(z), t\right) \geq M\left(T\left(y_{m}\right), T(z), \phi(r)\right) \geq M\left(G\left(y_{m}\right), G(z), r\right) \geq M\left(G\left(y_{m}\right), G(z), t\right)
$$

for all $t>0$ and $m \in \mathbb{N}_{0}$. Letting $m \rightarrow \infty$ in the above inequality, we get $T\left(y_{m}\right) \rightarrow T(z)$ as $m \rightarrow \infty$. By the uniqueness of the limit, we conclude that $G(z)=T(z)$ and $z$ is a coincidence point of $T$ and $G$.

Theorem 3.4 In addition to the hypotheses of Theorem 3.3, let $G$ be weakly compatible with $T$ if assumption (C2) holds. Suppose that for all coincidence points $y, v \in X$ of mappings $T$ and $G$, there exists $u \in X$ such that

(C3) $G(u)$ is comparable to $G(y)$ and $G(v)$;

(C4) $\lim _{t \rightarrow \infty} M(G(u), G(y), t)=\lim _{t \rightarrow \infty} M(G(u), G(v), t)=1$.

Then $T$ and $G$ have a unique common fixed point.

Proof Put $u_{0}=u$ and define a sequence $\left\{G\left(u_{m}\right)\right\}$ by $G\left(u_{m+1}\right)=T\left(u_{m}\right)$ for $m \in \mathbb{N}$. We may assume that $G(y) \preceq G\left(u_{0}\right)$ (the case $G(y) \succeq G\left(u_{0}\right)$ is treated similarly). Since $T$ is a $G$-isotone mapping, we have $G(y)=T(y) \preceq T\left(u_{0}\right)=G\left(u_{1}\right)$. By induction we obtain $G(y) \preceq G\left(u_{m}\right)$ for all $m \in \mathbb{N}_{0}$. Owing to $\lim _{t \rightarrow \infty} M(G(u), G(y), t)=1$, for any $\varepsilon \in(0,1)$, there exists $t_{2}>0$ such that $M\left(G\left(u_{0}\right), G(y), t_{2}\right)>1-\varepsilon$. Since $\phi \in \Phi_{w}$, there exists $t_{3} \geq t_{2}$ such that $\phi^{m}\left(t_{3}\right) \rightarrow 0$ as $m \rightarrow \infty$. Thus, for each $t>0$, there exists $m_{0} \in \mathbb{N}$ such that $\phi^{m}\left(t_{3}\right)<t$ for all $m \geq m_{0}$. So, by (9) and the monotonicity of $M(x, y, \cdot)$, we get for all $m \geq m_{0}$ and $t>0$,

$$
\begin{aligned}
M\left(G\left(u_{m}\right), G(y), t\right) & \geq M\left(G\left(u_{m}\right), G(y), \phi^{m}\left(t_{3}\right)\right) \\
& =M\left(T\left(u_{m-1}\right), T(y), \phi^{m}\left(t_{3}\right)\right) \\
& \geq M\left(G\left(u_{m-1}\right), G(y), \phi^{m-1}\left(t_{3}\right)\right) \\
& \geq \cdots \\
& \geq M\left(G\left(u_{0}\right), G(y), t_{3}\right) \\
& \geq M\left(G\left(u_{0}\right), G(y), t_{2}\right) \\
& >1-\varepsilon .
\end{aligned}
$$

Since $\varepsilon, t>0$ are arbitrary, we deduce that $M\left(G\left(u_{m}\right), G(y), t\right) \rightarrow 1$ as $m \rightarrow \infty$. This shows that $\lim _{m \rightarrow \infty} G\left(u_{m}\right)=G(y)$. Similarly, we find that $\lim _{m \rightarrow \infty} G\left(u_{m}\right)=G(v)$. The uniqueness of the limit proves that $G(y)=G(v)$. 
Denote $w=T(y)=G(y)$. Since $T$ and $G$ are weakly compatible mappings, we have $T(w)=$ $T G(y)=G T(y)=G(w)$. So, $w$ is also a coincidence point of $T$ and $G$. Therefore, $G(w)=$ $G(y)=w$ and $w$ is a common fixed point of $T$ and $G$. In order to prove the uniqueness, assume that $w^{*}$ is another common fixed point of $T$ and $G$. Then we have $w^{*}=G\left(w^{*}\right)=$ $G(w)=w$. This completes the proof.

Example 3.5 Let $(X, \preceq)$ be the partially ordered set with $X=[0,1]$ and the natural ordering $\leq$ of the real numbers as the partial ordering $\preceq$. Define $M: X \times X \times \mathbb{R}^{+} \rightarrow \mathbb{I}$ by

$$
M(x, y, t)= \begin{cases}0, & \text { if } t=0 \\ e^{-\frac{|x-y|}{t}}, & \text { if } t>0\end{cases}
$$

Then $M(x, y, \cdot): \mathbb{R}^{+} \rightarrow \mathbb{I}$ is continuous. Let $x * y=\min \{x, y\}$ for all $x, y \in X$. Then $(X, M, *)$ is a complete $F M S$ with $M(x, y, t) \rightarrow 1$ as $t \rightarrow \infty$, for all $x, y \in X$. Consider $T, G: X \rightarrow X$ defined by $T(x)=\frac{x^{2}}{3}+\frac{2}{3}$ and $G(x)=x$.

It is easy to verify the following statements.

(i) $T(X) \subseteq G(X)$ and $T$ is a $G$-isotone mapping.

(ii) The condition (C1) holds.

(iii) There exists $y_{0}=0$ such that $G\left(y_{0}\right)=0 \leq \frac{2}{3}=T\left(y_{0}\right)$.

Let $y, v \in X$ such that $G(y) \preceq G(v)$, that is, $y \leq v$. Next, we show that the inequality (9) is satisfied with $\phi(t)=\frac{3}{4} t$, for all $t>0$. If (9) does not hold, then there exists $t>0$ such that

$$
M\left(T(y), T(v), \frac{3}{4} t\right)<M(G(y), G(v), t)
$$

that is,

$$
e^{-\left|y^{2} / 3-v^{2} / 3\right| /(3 t / 4)}<e^{-|y-v| / t}
$$

that is,

$$
\frac{4}{9}\left|y^{2}-v^{2}\right|>|y-v|
$$

Since $y, v \in[0,1]$,

$$
|y-v|<\frac{4}{9}\left|y^{2}-v^{2}\right|=\frac{4}{9}|y-v|(y+v) \leq \frac{8}{9}|y-v|,
$$

which is impossible. Hence (9) holds.

By Theorems 3.3 and 3.4, $T$ and $G$ have a unique common fixed point, which is $z=1$. In this example, computing according to $z_{0}=G\left(y_{0}\right)$ and $z_{m+1}=G\left(y_{m+1}\right)=T\left(y_{m}\right)$ for every $m \in \mathbb{N}_{0}$, we obtain $\left\{z_{0}=0, z_{1}=\frac{2}{3}, z_{2}=\frac{22}{27}, z_{3}=\frac{1942}{2187}, \ldots\right\}$. Thus the sequence $\left\{z_{n}\right\}$ is a nontrivial sequence.

Example 3.6 Let $X=\{0,0.25,0.5,1.5,1.75,2\}$ and define $M: X \times X \times \mathbb{R}^{+} \rightarrow \mathbb{I}$ as follows:

$$
M(x, y, t)= \begin{cases}1, & \text { if }|x-y|<t \\ \frac{t}{|x-y|+t}, & \text { if }|x-y| \geq t\end{cases}
$$


As Gregori et al. have pointed out in [25], any $F M S(X, M)$ is equivalent to Menger space in the sense that $M(x, y, t)=F_{x, y}(t)$ for all $x, y \in X$ and $t \geq 0$. Thus, $(X, M)$ is a FMS under $*=\min$ (see Example in [8]).

Let $\tau_{M}$ be the discrete topology on $X$. Note that the metric space $(X, d)$ is complete, where $d$ is defined by $d(x, y)=|x-y|$. Next, we claim that if $\left\{x_{n}\right\}$ is a Cauchy sequence in $(X, M, *)$, then $\left\{x_{n}\right\}$ is also a Cauchy sequence in $(X, d)$. Indeed, if $\left\{x_{n}\right\}$ is not a Cauchy sequence in $(X, d)$, then there exist $\varepsilon_{0}>0$ and two sequences $\left\{n_{i}\right\}$ and $\left\{m_{i}\right\}$ such that $m_{i}>$ $n_{i} \geq i$ and $d\left(x_{m_{i}}, x_{n_{i}}\right) \geq \varepsilon_{0}$ for all $i \in \mathbb{N}$. Taking $\lambda_{0} \in\left(0, \frac{1}{2}\right]$, it follows from (13) that

$$
M\left(x_{m_{i}}, x_{n_{i}}, \varepsilon_{0}\right)=\frac{\varepsilon_{0}}{\varepsilon_{0}+\left|x_{m_{i}}-x_{n_{i}}\right|} \leq \frac{1}{2} \leq 1-\lambda_{0} \quad \text { for all } i \in \mathbb{N}
$$

which contradicts that $\left\{x_{n}\right\}$ is a Cauchy sequence in $(X, M, *)$. So, $\left\{x_{n}\right\}$ is also a Cauchy sequence in $(X, d)$. Since $(X, d)$ is complete, there exists $x \in X$ such that $x_{n} \rightarrow x$ as $n \rightarrow \infty$, and so for any $t>0$ there exists $n_{2} \in \mathbb{N}$ such that $\left|x_{n}-x\right|<t$ for all $n \geq n_{2}$. Thus, for any $t>0$ and $\varepsilon \in(0,1)$, by (13), we have $M\left(x_{n}, x, t\right)=1>1-\varepsilon$ for all $n \geq n_{2}$, which implies that $(X, M, *)$ is complete.

Endow $X$ with the following partial order:

$$
x, y \in X, x \preceq y \quad \Leftrightarrow \quad x=y \text { or }(x, y) \in\{(0,0.5),(0,0.25)\} .
$$

Let $\phi: \mathbb{R}^{+} \rightarrow \mathbb{R}^{+}$be defined by (2). Consider $T, G: X \rightarrow X$ defined by

$$
T(x)=\left\{\begin{array}{ll}
0, & \text { if } x \in\{0,0.25,0.5,1.75,2\}, \\
0.25, & \text { if } x=1.5 ;
\end{array} \quad G(x)= \begin{cases}0, & \text { if } x \in\{0,0.5\} \\
0.5, & \text { if } x=1.5 \\
0.25, & \text { if } x \in\{0.25,1.75,2\}\end{cases}\right.
$$

It is not difficult to prove the following statements.

(i) $T(X) \subseteq G(X)$.

(ii) The condition (C2) holds (since $\tau_{M}$ is the discrete topology on $X$ ).

(iii) There exists $y_{0}=0$ such that $G\left(y_{0}\right) \preceq T\left(y_{0}\right)$ and $\lim _{t \rightarrow \infty} M\left(G\left(y_{0}\right), T\left(y_{0}\right), t\right)=1$.

(iv) All conditions of Theorem 3.4 hold. In fact, $y=0$ and $v=0.5$ are all coincidence points of $T$ and $G$. Since $T G(0)=G T(0)$ and $T G(0.5)=G T(0.5)$, by Definition 2.20, $G$ is weakly compatible with $T$. In addition, there exists $u=1.5$ such that $G(y) \preceq G(u)$ and $G(v) \preceq G(u)$. It follows from (13) that (C4) holds.

(v) $T$ is a $G$-isotone mapping. Indeed, let $y, v \in X$ such that $G(y) \preceq G(v)$.

(a) If $G(y)=G(v)$ then $y=v$ or $y, v \in\{0,0.5\}$ or $y, v \in\{0.25,1.75,2\}$. Thus, $T(y)=T(v)$.

(b) If $(G(y), G(v))=(0,0.25)$, then $y \in\{0,0.5\}$ and $v \in\{0.25,1.75,2\}$. Thus, $T(y)=T(v)$.

(c) If $(G(y), G(v))=(0,0.5)$, then $y \in\{0,0.5\}$ and $v=1.5$. Thus, $(T(y), T(v))=(0,0.25)$.

Therefore, $T(y) \preceq T(v)$.

Next, we shall prove that (9) holds. Let $y, v \in X$ such that $G(y) \preceq G(v)$. It follows from (a) and (b) that $0=|T(y)-T(v)|<\phi(t)$ for all $t>0$. Thus, $M(T(y), T(v), \phi(t))=$ $1 \geq M(G(y), G(v), t)$ for all $t>0$, i.e., (9) holds. By (c), we have $|T(y)-T(v)|=0.25$ and 
$|G(y)-G(v)|=0.5$. If $\phi(t)>0.25$, by (13), it is evident that (9) holds. Suppose that $\phi(t) \leq$ 0.25 . From (2), it is easy to see that $\phi(t) \geq \frac{t}{1+t}$ for all $t \geq 0$. So, we have $t \leq \frac{1}{3}$. Therefore, $|G(y)-G(v)|>t$. By (13), we have

$$
M(T(y), T(v), \phi(t))=\frac{\phi(t)}{\frac{1}{4}+\phi(t)} \geq \frac{\frac{t}{1+t}}{\frac{1}{4}+\frac{t}{1+t}}=\frac{t}{t+\frac{1+t}{4}} \geq \frac{t}{t+\frac{1}{2}}=M(G(y), G(v), t)
$$

i.e., (9) holds.

By Theorems 3.3 and 3.4, $T$ and $G$ have a unique common fixed point, which is $z=0$. However, the totally ordered version of Theorems 3.3 and 3.4 cannot be applied to this example (since (9) does not hold). In fact, if $(G(y), G(v))=(0.5,0.25)$, then $y=1.5$ and $v \in$ $\{0.25,1.75,2\}$. Taking $t=\frac{7}{24}$, by (2), we have $\phi(t)=\frac{7}{31}<\frac{1}{4}=|T(y)-T(v)|$. By (13), we have

$$
M(T(y), T(v), \phi(t))=\frac{\phi(t)}{|T(y)-T(v)|+\phi(t)}=\frac{28}{59}<1=M(G(y), G(v), t) .
$$

So, (9) does not hold.

If $G$ is the identity mapping on $X$ in Theorems 3.3 and 3.4, then the following corollary is obtained immediately.

Corollary 3.7 Let $(X, M, *, \preceq)$ be a complete poFMS such that $*$ is a $t$-norm of H-type. Let $T: X \rightarrow X$ be a non-decreasing mapping. Assume that there exists $\phi \in \Phi_{w}$ such that, for all $t>0$ and $y, v \in X$ with $y \preceq v$,

$$
M(T(y), T(v), \phi(t)) \geq M(y, v, t) .
$$

Also suppose that either $T$ and $M(x, y, \cdot): \mathbb{R}^{+} \rightarrow \mathbb{I}$ are continuous or $\left(X, \tau_{M}, \preceq\right)$ has the sequential monotone property. If there exists $y_{0} \in X$, such that $y_{0} \asymp T\left(y_{0}\right)$ and $\lim _{t \rightarrow \infty} M\left(y_{0}, T\left(y_{0}\right), t\right)=1$, then $T$ has a fixed point.

Furthermore, suppose that for all fixed points $y, v \in X$ of $T$, there exists $u \in X$ such that $u$ is comparable to $y$ and $v$ and $\lim _{t \rightarrow \infty} M(u, y, t)=\lim _{t \rightarrow \infty} M(u, v, t)=1$. Then $T$ has a unique fixed point.

Remark 3.8 Corollary 3.7 can be considered as a partially ordered version of Theorem 4.1 in $[10]$.

Example 3.9 Let $(X, M, \preceq, *), T, \phi$ be the same as in Example 3.6. Using a similar argument to Example 3.6, we deduce that the conditions of Corollary 3.7 are satisfied. So, $T$ has a unique fixed point, which is $z=0$. However, Theorem $4.1 \mathrm{in}$ [10] cannot be applied to this example because the condition ' $M(T(y), T(v), \phi(t)) \geq M(y, v, t)$ for all $y, v \in X$ and $t>0$ ' does not hold. In fact, if $(y, v)=(1.75,1.5)$, then $|T(y)-T(v)|=\frac{1}{4}$. Taking $t=\frac{1}{4}$, by (2), we have $\phi(t)=\frac{1}{5}$. Thus, by (13), we have

$$
M(T(y), T(v), \phi(t))=\frac{\phi(t)}{|T(y)-T(v)|+\phi(t)}=\frac{4}{9}<\frac{1}{2}=M(y, v, t) .
$$

Next, we give some basic concepts and results that we will need to obtain some coupled and multidimensional fixed point results. 
Definition 3.10 Let $F: X^{n} \rightarrow X$ and $g: X \rightarrow X$ be two mappings. A point $\left(x_{1}, x_{2}, \ldots, x_{n}\right) \in$ $X^{n}$ is a common fixed point of $F$ and $g$ if $F\left(x_{\sigma_{i}(1)}, x_{\sigma_{i}(2)}, \ldots, x_{\sigma_{i}(n)}\right)=g x_{i}=x_{i}$ for $i \in \Lambda_{n}$.

Definition 3.11 Given $n \geq 2$, the mappings $F: X^{n} \rightarrow X$ and $g: X \rightarrow X$ are weakly compatible (or the pair $(F, g)$ is $w$-compatible) if

$$
\begin{aligned}
& F\left(x_{\sigma_{i}(1)}, x_{\sigma_{i}(2)}, \ldots, x_{\sigma_{i}(n)}\right)=g x_{i}, \quad \forall i \in \Lambda_{n} \\
& \quad \Rightarrow \quad g F\left(x_{\sigma_{i}(1)}, x_{\sigma_{i}(2)}, \ldots, x_{\sigma_{i}(n)}\right)=F\left(g x_{\sigma_{i}(1)}, g x_{\sigma_{i}(2)}, \ldots, g x_{\sigma_{i}(n)}\right), \quad \forall i \in \Lambda_{n} .
\end{aligned}
$$

Lemma 3.12 Let $(X, M, *)$ be a FMS such that $*$ is a continuous $t$-norm. Let $M^{n}: X^{n} \times$ $X^{n} \times \mathbb{R}^{+} \rightarrow \mathbb{I}$ be given by

$$
M^{n}(A, B, t)=*_{i=1}^{n} M\left(a_{i}, b_{i}, t\right)
$$

for all $A=\left(a_{1}, a_{2}, \ldots, a_{n}\right), B=\left(b_{1}, b_{2}, \ldots, b_{n}\right) \in X^{n}$, and all $t \geq 0$. Then the following properties hold:

(i) $\left(X^{n}, M^{n}, *\right)$ is also a FMS.

(ii) Let $\left\{A_{m}=\left(a_{m}^{1}, a_{m}^{2}, \ldots, a_{m}^{n}\right)\right\}$ be a sequence on $X^{n}$ and let $A=\left(a_{1}, a_{2}, \ldots, a_{n}\right) \in X^{n}$.

Then $\left\{A_{m}\right\} \rightarrow A$ if, and only if, $\left\{a_{m}^{i}\right\} \rightarrow a_{i}$ for all $i \in\{1,2, \ldots, n\}$.

(iii) If $(X, M, *)$ is complete, then $\left(X^{n}, M^{n}, *\right)$ is complete.

Proof The proofs of (i) and (ii) in Lemma 3.12 are the same as Lemma 13 in [15]. Next, we shall prove that (iii) holds. Suppose that $\left\{Y_{m}\right\}$ is a Cauchy sequence in $\left(X^{n}, M^{n}, *\right)$. Thus, for any $\varepsilon \in(0,1)$ and $t>0$, there exists $n_{3} \in \mathbb{N}$ such that $M^{n}\left(Y_{n}, Y_{m}, t\right)>1-\varepsilon$ for all $n, m \geq n_{3}$. By (14), we have

$$
\min _{1 \leq i \leq n} M\left(y_{n}^{i}, y_{m}^{i}, t\right) \geq *_{i=1}^{n} M\left(y_{n}^{i}, y_{m}^{i}, t\right)>1-\varepsilon \quad \text { for all } n, m \geq n_{3} .
$$

Thus, for $n, m \geq n_{3}$, we have $M\left(y_{n}^{i}, y_{m}^{i}, t\right)>1-\varepsilon$ for each $i \in \Lambda_{n}$. Therefore, $\left\{y_{m}^{i}\right\}$ is a Cauchy sequence in $(X, M, *)$ for each $i \in \Lambda_{n}$. Since $(X, M, *)$ is complete, then $\left\{y_{m}^{i}\right\}$ converges to a point $y_{i}$ of $X$ for each $i \in \Lambda_{n}$. Thus, $\left\{Y_{m}\right\}$ converges to a point $Y$ of $X^{n}$. That is, $\left(X^{n}, M^{n}, *\right)$ is complete. This completes the proof.

The following multidimensional fixed point theorem is an immediate consequence of Theorems 3.3 and 3.4.

Theorem 3.13 Let $(X, M, *, \preceq)$ be a complete poFMS with a continuous $t$-norm $*$ of $H$ type. Let $\Phi=\left(\sigma_{1}, \sigma_{2}, \ldots, \sigma_{n}\right)$ be an n-tuple of mappings from $\Lambda_{n}$ into itself verifying $\sigma_{i} \in \Omega_{A, B}$ if $i \in A$ and $\sigma_{i} \in \Omega_{A, B}^{\prime}$ if $i \in B$. Suppose that $F: X^{n} \rightarrow X$ and $g: X \rightarrow X$ are two mappings such that $F$ has the mixed $g$-monotone property on $X$ and $F\left(X^{n}\right) \subseteq g(X)$. Assume that there exists $\phi \in \Phi_{w}$ such that

$$
M\left(F\left(x_{1}, x_{2}, \ldots, x_{n}\right), F\left(y_{1}, y_{2}, \ldots, y_{n}\right), \phi(t)\right) \geq \gamma\left(*_{i=1}^{n} M\left(g x_{i}, g y_{i}, t\right)\right)
$$

for all $t>0$ and all $x_{1}, x_{2}, \ldots, x_{n}, y_{1}, y_{2}, \ldots, y_{n} \in X$ with $g x_{i} \preceq_{i}$ gy for $i \in \Lambda_{n}$, where $\gamma$ : $[0,1] \rightarrow[0,1]$ is a mapping such that $*^{n} \gamma(a) \geq$ a for each $a \in[0,1]$. Suppose that

$$
\gamma\left(*_{i=1}^{n} M\left(g x_{\sigma_{j}(i)}, g y_{\sigma_{j}(i)}, t\right)\right) \geq \gamma\left(*_{i=1}^{n} M\left(g x_{i}, g y_{i}, t\right)\right) \quad \text { for } j \in \Lambda_{n},
$$


for all $x_{1}, x_{2}, \ldots, x_{n}, y_{1}, y_{2}, \ldots, y_{n} \in X$ with $g x_{i} \preceq_{i} g y_{i}$ for $i \in \Lambda_{n}$. Suppose that either

(C5) $F$ and $g$ are continuous and $\Phi$-compatible and $M(x, y, \cdot): \mathbb{R}^{+} \rightarrow \mathbb{I}$ is continuous, or

(C6) $\left(X, \tau_{M}, \preceq\right)$ has the sequential monotone property and $g(X)$ is closed.

If there exist $x_{0}^{1}, x_{0}^{2}, \ldots, x_{0}^{n} \in X$ satisfying

$$
\begin{aligned}
& g x_{0}^{i} \preceq_{i} F\left(x_{0}^{\sigma_{i}(1)}, x_{0}^{\sigma_{i}(2)}, \ldots, x_{0}^{\sigma_{i}(n)}\right) \quad \text { and } \\
& \lim _{t \rightarrow \infty} M\left(g x_{0}^{i}, F\left(x_{0}^{\sigma_{i}(1)}, x_{0}^{\sigma_{i}(2)}, \ldots, x_{0}^{\sigma_{i}(n)}\right), t\right)=1
\end{aligned}
$$

for $i \in \Lambda_{n}$, then $F$ and $g$ have, at least, one $\Upsilon$-coincidence point.

Furthermore, assume that for all pairs of $\Upsilon$-coincidence points $\left(x_{1}, x_{2}, \ldots, x_{n}\right),\left(y_{1}, y_{2}\right.$, $\left.\ldots, y_{n}\right) \in X^{n}$ of $F$ and $g$ there exists $\left(u_{1}, u_{2}, \ldots, u_{n}\right) \in X^{n}$ such that

(C7) $\left(g u_{1}, g u_{2}, \ldots, g u_{n}\right)$ is comparable to $\left(g x_{1}, g x_{2}, \ldots, g x_{n}\right)$ and $\left(g y_{1}, g y_{2}, \ldots, g y_{n}\right)$;

(C8) $\lim _{t \rightarrow \infty} M\left(g u_{i}, g x_{i}, t\right)=\lim _{t \rightarrow \infty} M\left(g u_{i}, g y_{i}, t\right)=1$ for $i \in \Lambda_{n}$.

Also, assume that $F$ is weakly compatible with $g$ if assumption (C6) holds. Then $F$ and $g$ have a unique common fixed point.

Proof Since $(X, M, *, \preceq)$ is a complete poFMS such that $*$ is a continuous $t$-norm of $H$ type, so is $\left(X^{n}, M^{n}, *, \preceq_{n}\right)$ by Lemma 3.12. Let $T: X^{n} \rightarrow X^{n}$ and $G: X^{n} \rightarrow X^{n}$ be two mappings defined by

$$
\begin{aligned}
T(Y)= & \left(F\left(y_{\sigma_{1}(1)}, y_{\sigma_{1}(2)}, \ldots, y_{\sigma_{1}(n)}\right), \ldots, F\left(y_{\sigma_{i}(1)}, y_{\sigma_{i}(2)}, \ldots, y_{\sigma_{i}(n)}\right), \ldots,\right. \\
& \left.F\left(y_{\sigma_{n}(1)}, y_{\sigma_{n}(2)}, \ldots, y_{\sigma_{n}(n)}\right)\right)
\end{aligned}
$$

and

$$
G(Y)=\left(g y_{1}, g y_{2}, \ldots, g y_{n}\right)
$$

for $Y \in X^{n}$. It follows from $F\left(X^{n}\right) \subseteq g(X)$ that $T\left(X^{n}\right) \subseteq G\left(X^{n}\right)$. By (14), (17) and the continuity of $*$, there exists $X_{0}$ such that $G\left(X_{0}\right) \preceq_{n} T\left(X_{0}\right)$ and $\lim _{t \rightarrow \infty} M^{n}\left(G\left(X_{0}\right), T\left(X_{0}\right), t\right)=1$.

Suppose that $\left\{Y_{m}\right\}_{m \geq 0} \subset X^{n}$ such that $\left\{G\left(Y_{m}\right)\right\}_{m \geq 0}$ is monotone and the following limit exists: $\lim _{m \rightarrow \infty} T\left(Y_{m}\right)=\lim _{m \rightarrow \infty} G\left(Y_{m}\right) \in X^{n}$. From (18) and (19), we see that, for sequences $\left\{y_{m}^{1}\right\}_{m \geq 0},\left\{y_{m}^{2}\right\}_{m \geq 0}, \ldots,\left\{y_{m}^{n}\right\}_{m \geq 0} \subset X$ such that $\left\{g y_{m}^{1}\right\}_{m \geq 0},\left\{g y_{m}^{2}\right\}_{m \geq 0}, \ldots,\left\{g y_{m}^{n}\right\}_{m \geq 0}$ are monotone and the following limit exists: for all $i \in \Lambda_{n}, \lim _{m \rightarrow \infty} F\left(y_{m}^{\sigma_{i}(1)}, y_{m}^{\sigma_{i}(2)}, \ldots, y_{m}^{\sigma_{i}(n)}\right)=$ $\lim _{m \rightarrow \infty} g y_{m}^{i} \in X$. Since $F$ and $g$ are $\Phi$-compatible, we have

$$
\begin{aligned}
\lim _{m \rightarrow \infty} M^{n}\left(G T\left(Y_{m}\right), T G\left(Y_{m}\right), t\right)= & *_{i=1}^{n} \lim _{m \rightarrow \infty} M\left(g F\left(y_{m}^{\sigma_{i}(1)}, y_{m}^{\sigma_{i}(2)}, \ldots, y_{m}^{\sigma_{i}(n)}\right),\right. \\
& \left.F\left(g y_{m}^{\sigma_{i}(1)}, g y_{m}^{\sigma_{i}(2)}, \ldots, g y_{m}^{\sigma_{i}(n)}\right), t\right)=1 .
\end{aligned}
$$

Therefore, $T$ and $G$ are compatible.

Now, we show that $T$ is a $G$-isotone mapping. Suppose that $G(Y) \preceq_{n} G(V)$ for $Y, V \in X^{n}$. By (1) and (19), we have $g y_{j} \preceq g v_{j}$ when $j \in A$ and $g y_{j} \succeq g v_{j}$ when $j \in B$. For each $i \in A$, we have $\sigma_{i} \in \Omega_{A, B}$. So, for fixed $i \in A$, we have $g y_{\sigma_{i}(j)} \preceq g v_{\sigma_{i}(j)}$ when $j \in A$ and $g y_{\sigma_{i}(j)} \succeq g v_{\sigma_{i}(j)}$ when $j \in B$. Thus, by the mixed $g$-monotone property of $F$, for fixed $i \in A$, we have

$$
\begin{aligned}
& F\left(y_{\sigma_{i}(1)}, \ldots, y_{\sigma_{i}(j-1)}, y_{\sigma_{i}(j)}, y_{\sigma_{i}(j+1)}, \ldots, y_{\sigma_{i}(n)}\right) \\
& \quad \preceq F\left(y_{\sigma_{i}(1)}, \ldots, y_{\sigma_{i}(j-1)}, v_{\sigma_{i}(j)}, y_{\sigma_{i}(j+1)}, \ldots, y_{\sigma_{i}(n)}\right)
\end{aligned}
$$


when $j \in A$. Similarly, if $j \in B$, then inequality (20) holds for fixed $i \in A$. So, for fixed $i \in A$, inequality (20) holds for all $j$. From this, we have

$$
\begin{aligned}
F\left(y_{\sigma_{i}(1)}, y_{\sigma_{i}(2)}, \ldots, y_{\sigma_{i}(n)}\right) & \preceq F\left(v_{\sigma_{i}(1)}, y_{\sigma_{i}(2)}, y_{\sigma_{i}(3)}, \ldots, y_{\sigma_{i}(n)}\right) \\
& \preceq F\left(v_{\sigma_{i}(1)}, v_{\sigma_{i}(2)}, y_{\sigma_{i}(3)}, \ldots, y_{\sigma_{i}(n)}\right) \\
& \preceq \ldots \\
& \preceq F\left(v_{\sigma_{i}(1)}, v_{\sigma_{i}(2)}, \ldots, v_{\sigma_{i}(n)}\right)
\end{aligned}
$$

for $i \in A$. Similarly, we have

$$
F\left(y_{\sigma_{i}(1)}, y_{\sigma_{i}(2)}, \ldots, y_{\sigma_{i}(n)}\right) \succeq F\left(v_{\sigma_{i}(1)}, v_{\sigma_{i}(2)}, \ldots, v_{\sigma_{i}(n)}\right)
$$

for $i \in B$. Thus, by (18), (21), and (22), we deduce that $T$ is a $G$-isotone mapping.

The conditions (C7) and (C8) imply that (C3) and (C4) hold w.r.t. $\left(X^{n}, M^{n}, *, \preceq_{n}\right)$. It is easy to deduce that $T$ and $G$ are weakly compatible if assumption (C2) holds w.r.t. $\left(X^{n}, M^{n}, *, \preceq_{n}\right)$. If $F$ and $g$ are continuous, then $T$ and $G$ are continuous.

Given $G(Y) \preceq_{n} G(V)$, by Proposition 2.1, $\left(g y_{\sigma_{i}(1)}, g y_{\sigma_{i}(2)}, \ldots, g y_{\sigma_{i}(n)}\right)$ and $\left(g v_{\sigma_{i}(1)}, g v_{\sigma_{i}(2)}, \ldots\right.$, $\left.g v_{\sigma_{i}(n)}\right)$ are comparable by $\preceq_{n}$. Therefore, (15) and (16) can be applied to these points, and it follows that for all $t>0$,

$$
\begin{aligned}
M^{n}(T(Y), T(V), \phi(t)) & =*_{i=1}^{n} M\left(F\left(y_{\sigma_{i}(1)}, y_{\sigma_{i}(2)}, \ldots, y_{\sigma_{i}(n)}\right), F\left(v_{\sigma_{i}(1)}, v_{\sigma_{i}(2)}, \ldots, v_{\sigma_{i}(n)}\right), \phi(t)\right) \\
& \geq *_{i=1}^{n} \gamma\left(*_{j=1}^{n} M\left(g y_{\sigma_{i}(j)}, g v_{\sigma_{i}(j)}, t\right)\right) \\
& \geq *_{i=1}^{n} \gamma\left(*_{j=1}^{n} M\left(g y_{j}, g v_{j}, t\right)\right) \\
& =*_{i=1}^{n} \gamma\left(M^{n}(G(Y), G(V), t)\right) \\
& =*^{n} \gamma\left(M^{n}(G(Y), G(V), t)\right) \\
& \geq M^{n}(G(Y), G(V), t) .
\end{aligned}
$$

Next we shall prove that the condition (C2) of Theorem 3.3 holds w.r.t. $\left(X^{n}, M^{n}, *, \preceq_{n}\right)$. Since $g(X)$ is closed, so is $G(X)$. Suppose that $\left\{Z_{m}\right\}$ is a non-decreasing sequence in $X^{n}$ such that $Z_{m} \rightarrow Z(m \rightarrow \infty)$. Using Lemma 3.12, we have $z_{m}^{i} \rightarrow z^{i}(m \rightarrow \infty)$ for all $i \in \Lambda_{n}$. Since $Z_{m} \preceq_{n} Z_{m+1}$ for all $m \in \mathbb{N}_{0}$, then $\left(z_{m}^{i}\right)_{m \in \mathbb{N}_{0}}$ is a non-decreasing sequence when $i \in A$ and $\left(z_{m}^{i}\right)_{m \in \mathbb{N}_{0}}$ is a non-increasing sequence when $i \in B$. If $i \in A$, as $\left(X, \tau_{M}, \preceq\right)$ has the sequential monotone property, then we have $z_{m}^{i} \preceq z^{i}$ for all $m \in \mathbb{N}_{0}$. Similarly, if $i \in B$, then $z_{m}^{i} \succeq z^{i}$ for all $m \in \mathbb{N}_{0}$. That is, $Z_{m} \preceq_{n} Z$ for every $m \in \mathbb{N}_{0}$. The other case is treated similarly.

Therefore, all conditions of Theorems 3.3 and 3.4 hold w.r.t. $\left(X^{n}, M^{n}, *, \preceq_{n}\right)$. Theorem 3.3 implies that $T$ and $G$ have a coincidence point, which is a $\Upsilon$-coincidence point of $F$ and $g$. Moreover, it follows from Theorem 3.4 that $T$ and $G$ have a unique common fixed point, which is a unique common fixed point of $F$ and $g$.

Remark 3.14 Theorem 3.13 improves Theorems 18 and 26 in [7]:

(i) We use $\phi \in \Phi_{w}$, and $\Phi_{w}$ is a class of more general functions than $\phi(t)=k t, k \in(0,1)$.

(ii) The continuity of $g$ and $\Phi$-compatible of $F$ and $g$ are omitted if assumption (C6) holds. The continuity of $\gamma$ is not necessary. 
(iii) The condition $\lim _{t \rightarrow \infty} M(x, y, t)=1$ for all $x, y \in X$ is weakened by conditions (17) and (C8).

(iv) Our result is valid for fuzzy metric spaces in the sense of Kramosil and Michálek, so it is also valid for fuzzy metric spaces in the sense of George and Veeramani. The completeness is a weaker kind of completeness in Theorem 3.13 (see [26]).

Taking $n=2, A=\{1\}$, and $B=\{2\}$ in Theorem 3.13, we deduce the following coupled fixed point theorem improving Theorem 3.1 in [6].

Corollary 3.15 Let $(X, M, *, \preceq)$ be a complete poFMS such that $*$ is a continuous $t$-norm of $H$-type. Let $F: X^{2} \rightarrow X$ and $g: X \rightarrow X$ be two mappings such that $F$ has the mixed $g$-monotone property on $X$ and $F\left(X^{2}\right) \subseteq g(X)$. Assume that there exists $\phi \in \Phi_{w}$ such that

$$
M\left(F\left(x_{1}, x_{2}\right), F\left(y_{1}, y_{2}\right), \phi(t)\right) \geq \gamma\left(M\left(g\left(x_{1}\right), g\left(y_{1}\right), t\right) * M\left(g\left(x_{2}\right), g\left(y_{2}\right), t\right)\right)
$$

for all $t>0$ and $x_{1}, x_{2}, y_{1}, y_{2} \in X$ with $g\left(x_{1}\right) \preceq g\left(y_{1}\right)$ and $g\left(x_{2}\right) \succeq g\left(y_{2}\right)$, where $\gamma:[0,1] \rightarrow$ $[0,1]$ is a mapping such that $\gamma(a) * \gamma(a) \geq a$ for each $a \in[0,1]$. Suppose that either $F$ and $g$ are continuous and compatible and $M(x, y, \cdot): \mathbb{R}^{+} \rightarrow \mathbb{I}$ is continuous or condition (C6) holds. If there are $x_{0}^{1}, x_{0}^{2} \in X$ such that $g\left(x_{0}^{1}\right) \preceq F\left(x_{0}^{1}, x_{0}^{2}\right), g\left(x_{0}^{2}\right) \succeq F\left(x_{0}^{2}, x_{0}^{1}\right)$ and $\lim _{t \rightarrow \infty} M\left(g\left(x_{0}^{1}\right), F\left(x_{0}^{1}, x_{0}^{2}\right), t\right)=\lim _{t \rightarrow \infty} M\left(g\left(x_{0}^{2}\right), F\left(x_{0}^{2}, x_{0}^{1}\right), t\right)=1$, then $F$ and $g$ have a coupled coincidence point in $X$.

Furthermore, assume that for all pairs of coupled coincidence points $\left(x_{1}, x_{2}\right),\left(y_{1}, y_{2}\right) \in X^{2}$ of $F$ and $g$ there exists $\left(u_{1}, u_{2}\right) \in X^{2}$ such that $\left(g u_{1}, g u_{2}\right)$ is comparable to $\left(g x_{1}, g x_{2}\right)$ and $\left(g y_{1}, g y_{2}\right), \lim _{t \rightarrow \infty} M\left(g u_{i}, g x_{i}, t\right)=\lim _{t \rightarrow \infty} M\left(g u_{i}, g y_{i}, t\right)=1$ for $i \in \Lambda_{2}$. Also, assume that $F$ are weakly compatible with $g$ if assumption (C6) holds. Then $F$ and $g$ have a unique common coupled fixed point.

Remark 3.16 Corollary 3.15 is better than Theorem 3.2 in [5] in two senses.

(i) In Corollary 3.15, we use $\phi \in \Phi_{w}$, and $\Phi_{w}$ is a class of more general functions than $\Phi$ in Theorem 3.2 of [5].

(ii) Corollary 3.15 is valid for partially ordered fuzzy metric spaces in the sense of Kramosil and Michálek, so it is also valid for fuzzy metric spaces in the sense of George and Veeramani.

\section{Competing interests}

The author declares that she has no competing interests.

\section{Author's contributions}

SW completed the paper herself. The author read and approved the final manuscript.

\section{Acknowledgements}

The author thanks the editor and the referees for their useful comments and suggestions. Supported by the Natural Science Foundation of Jiangsu Province under grant (13KJB110028).

Received: 18 August 2015 Accepted: 9 October 2015 Published online: 23 December 2015

\section{References}

1. Guo, D, Lakshmikantham, V: Coupled fixed points of nonlinear operators with applications. Nonlinear Anal. 11, 623-632 (1987)

2. Bhaskar, TG, Lakshmikantham, V: Fixed point theorems in partially ordered metric spaces and applications. Nonlinear Anal. 65, 1379-1393 (2006)

3. Sedghi, S, Altun, I, Shobe, N: Coupled fixed point theorems for contractions in fuzzy metric spaces. Nonlinear Anal. 72, 1298-1304 (2010) 
4. Hu, XQ: Common coupled fixed point theorems for contractive mappings in fuzzy metric spaces. Fixed Point Theory Appl. 2011, 363716 (2011). doi:10.1155/2011/363716

5. Hu, XQ, Zheng, MX, Damjanović, B, Shao, XF: Common coupled fixed point theorems for weakly compatible mappings in fuzzy metric spaces. Fixed Point Theory Appl. 2013, 220 (2013). doi:10.1186/1687-1812-2013-220

6. Choudhury, BS, Das, K, Das, P: Coupled coincidence point results for compatible mappings in partially ordered fuzzy metric spaces. Fuzzy Sets Syst. 222, 84-97 (2013)

7. Roldán, A, Martínez-Moreno, J, Roldán, C, Cho, YJ: Multidimensional coincidence point results for compatible mappings in partially ordered fuzzy metric spaces. Fuzzy Sets Syst. 251(16), 71-82 (2014)

8. Ćirić, L: Solving the Banach fixed point principle for nonlinear contractions in probabilistic metric spaces. Nonlinear Anal. 72, 2009-2018 (2010)

9. Jachymski, J: On probabilistic $\phi$-contractions on Menger spaces. Nonlinear Anal. 73, 2199-2203 (2010)

10. Fang, JX: On $\varphi$-contractions in probabilistic and fuzzy metric spaces. Fuzzy Sets Syst. 267, $86-99$ (2015)

11. Roldán, A, Martínez-Moreno, J, Roldán, C: Multidimensional fixed point theorems in partially ordered metric spaces. J. Math. Anal. Appl. 396, 536-545 (2012)

12. Roldán, A, Martínez-Moreno, J, Roldán, C, Karapınar, E: Some remarks on multidimensional fixed point theorems. Fixed Point Theory 15(2), 545-558 (2014)

13. Wang, S: Coincidence point theorems for G-isotone mappings in partially ordered metric spaces. Fixed Point Theory Appl. 2013, 96 (2013). doi:10.1186/1687-1812-2013-96

14. Lakshmikantham, V, Ćirić, L: Coupled fixed point theorems for nonlinear contractions in partially ordered metric spaces. Nonlinear Anal. 70, 4341-4349 (2009)

15. Francisco, A, Hierro, RL, Sintunavarat, W: Common fixed point theorems in fuzzy metric spaces using the CLRg property. In: Fuzzy Sets Syst (2014). doi:10.1016/j.fss.2014.11.005

16. Karapınar, E, Roldán, A, Martinez-Moreno, J, Roldán, C: Meir-Keeler type multidimensional fixed point theorems in partially ordered metric spaces. Abstr. Appl. Anal. 2013, Article ID 406026 (2013)

17. Ćirić, L, Abbas, M, Damjanovic, B, Saadati, R: Common fuzzy fixed point theorems in ordered metric spaces. Math. Comput. Model. 53, 1737-1741 (2011)

18. Schweizer, B, Sklar, A: Probabilistic Metric Spaces. Dover, New York (2005)

19. Hadžić, O, Pap, E: Fixed Point Theory in Probabilistic Metric Spaces. Kluwer Academic, Dordrecht (2001)

20. Kramosil, I, Michálek, J: Fuzzy metrics and statistical metric spaces. Kybernetika 11, 336-344 (1975)

21. George, A, Veeramani, P: On some result in fuzzy metric space. Fuzzy Sets Syst. 64, 395-399 (1994)

22. Grabiec, M: Fixed points in fuzzy metric spaces. Fuzzy Sets Syst. 27(3), 385-389 (1988)

23. Abbas, M, Khan, MA, Radenovic, S: Common coupled fixed point theorems in cone metric spaces for $w$-compatible mappings. Appl. Math. Comput. 217(1), 195-202 (2010)

24. Rodríguez-López, J, Romaguera, S: The Hausdorff fuzzy metric on compact sets. Fuzzy Sets Syst. 147, 273-283 (2004)

25. Gregori, V, Morillas, S, Sapena, A: On a class of completable fuzzy metric spaces. Fuzzy Sets Syst. 161, 2193-2205 (2010)

26. Vasuki, R, Veeramani, P: Fixed point theorems and Cauchy sequences in fuzzy metric spaces. Fuzzy Sets Syst. 135, 415-417 (2003)

\section{Submit your manuscript to a SpringerOpen ${ }^{\circ}$ journal and benefit from:}

- Convenient online submission

Rigorous peer review

- Immediate publication on acceptance

- Open access: articles freely available online

- High visibility within the field

- Retaining the copyright to your article 